\title{
A Computer-Assisted Investigation of Ramanujan Pairs
}

\author{
By Richard Blecksmith, John Brillhart and Irving Gerst
}

Dedicated to D. H. Lehmer on his eightieth birthday

\begin{abstract}
Four new Ramanujan pairs $\left\{a_{i}\right\},\left\{b_{j}\right\}$ are given along with the theorem that no such pairs exist with $a_{1}=1$ and $a_{2}=s$ for any $s \geqslant 5$. All finite Ramanujan pairs are determined and their significance in bounding the local branching degree in the search tree for such pairs is discussed. The search techniques and programs that were used are also described. The parity of the coefficients in the power series is determined in two of the new identities. Partition interpretations of the six recent identities are also given.
\end{abstract}

1. Introduction. (a) By a Ramanujan Pair (R.P.) we mean two infinite, increasing sequences of positive integers $\left\{a_{i}\right\},\left\{b_{j}\right\}$ for which (1) formally holds when the two sides are expanded into Maclaurin series:

$$
\prod_{i=1}^{\infty}\left(1-x^{a_{i}}\right)^{-1}=1+\sum_{j=1}^{\infty} x^{b_{j}} /(1-x)\left(1-x^{2}\right) \cdots\left(1-x^{j}\right) . *
$$

This definition is essentially the same as the one given by Andrews [3], except that here we use a single letter for the numerator exponent on the right side of (1) and explicitly require that the sequence $\left\{a_{i}\right\}$ be increasing. Also in [3], Andrews conjectured that the only R.P.'s were those listed in that paper. (These are entries 1-4 in Table 1 below.) Shortly thereafter M. Hirschhorn [9] showed that this listing was actually incomplete by finding two more pairs - numbers 5 and 6 in Table 1.

In our investigation we have discovered four more R.P.'s-entries 7-10 in Table 1 - using a Fortran search program written for the Data General Eclipse S/230 at the Mathematics Department of the University of Arizona. (For the initial announcement of these results see Notices Amer. Math. Soc., Aug. 1982, p. 390.) As it happens, these four new pairs, as well as the two pairs of Hirschhorn, can easily be derived from six identities that have appeared previously in the literature, but in a form that disguises their connection with R.P.'s. In fact, the identities numbered 86, $83,94,98,96$, and 99 in Slater [13] directly yield the identities associated with the pairs 5-10 when the general term on their right sides is written as a sum of two

Received April 1, 1985; revised July 15, 1985.

1980 Mathematics Subject Classification. Primary 10A45, 05-04, 05 A19.

Key words and phrases. Ramanujan pairs, computer search tree.

* This also holds as a functional equation for $|x|<1$. 
TABLE 1

Ramanujan Pairs

$$
\left\{a_{n}\right\}, n \geqslant 1
$$

$(m \geqslant 1)$

$\left\{b_{n}\right\}, n \geqslant 1$

1. $\{m, m+1, m+2, \ldots\}$

$b_{n}=n m$

2. $\{m, m+1, m+2, \ldots, 2 m-1,2 m+1,2 m+3, \ldots\}$

$b_{n}=n m+\frac{n(n-1)}{2}$

3. $\{m \equiv \pm 1(\bmod 5)\}$

$b_{n}=n^{2}$

4. $\{m \equiv \pm 2(\bmod 5)\}$

$b_{n}=n(n+1)$

5. $\{m \equiv \pm 1, \pm 4, \pm 6, \pm 7(\bmod 16)\}$

$\left\{\begin{array}{l}b_{2 n-1}=2 n^{2}-1 \\ b_{2 n}=2 n(n+1)\end{array}\right.$

6. $\{m \equiv \pm 2, \pm 3, \pm 4, \pm 5(\bmod 16)\}$

$\left\{\begin{array}{l}b_{2 n-1}=2 n^{2} \\ b_{2 n}=2 n(n+1)\end{array}\right.$

7. $\{m \equiv \pm 1, \pm 2, \pm 5, \pm 6, \pm 8, \pm 9(\bmod 20)\}$ $\left\{\begin{array}{l}b_{2 n-1}=n(n+1)-1 \\ b_{2 n}=n(n+1)\end{array}\right.$

8. $\{m \equiv \pm 1, \pm 3, \pm 4, \pm 5, \pm 7, \pm 9(\bmod 20)\}$ $\left\{\begin{array}{l}b_{2 n-1}=n^{2} \\ b_{2 n}=n(n+2)\end{array}\right.$

9. $\{m \equiv \pm 1, \pm 3, \pm 5, \pm 7, \pm 8, \pm 9(\bmod 20)\}$ $\left\{\begin{array}{l}b_{2 n-1}=n(n+2)-2 \\ b_{2 n}=n(n+2)\end{array}\right.$

10. $\{m \equiv \pm 2, \pm 3, \pm 4, \pm 5, \pm 6, \pm 7(\bmod 20)\}$ $\left\{\begin{array}{l}b_{2 n-1}=n(n+1) \\ b_{2 n}=n(n+3) .\end{array}\right.$

fractions in an appropriate way. For example, identity 94 yields the identity associated with pair 7 by writing

$$
\begin{aligned}
\frac{x^{k(k+1)}}{(1-x)\left(1-x^{2}\right) \cdots\left(1-x^{2 k+1}\right)}= & \frac{x^{k(k+1)}}{(1-x) \cdots\left(1-x^{2 k}\right)} \\
& +\frac{x^{k^{2}+3 k+1}}{(1-x) \cdots\left(1-x^{2 k}\right)\left(1-x^{2 k+1}\right)} .
\end{aligned}
$$

(It should be mentioned that the $\left\{a_{i}\right\}$ sequences in pairs 5-10 appear in [11, p. 57] as well.)

Although an R.P. in [3] is defined only for infinite sequences, we will discuss in Section 2 the question whether or not an equation of form (1) can ever be satisfied if one or both of $\left\{a_{i}\right\},\left\{b_{j}\right\}$ is finite. There we will find all such pairs and interpret their significance in terms of the structure of the search trees of possible new R.P.'s.

In Section 3, we detail the search method we have developed, which creates and examines the search tree associated with the given starting values. The complete search tree for the starting values $a_{1}=1, a_{2}=6$ is given in Figure 11, both to illustrate our presentation of a search tree and to give the details to complete the nonexistence proof presented in Section 5.

Section 4 contains the details of the various search programs we have used and the algorithms on which they are based. 
Section 5 contains a nonexistence theorem stating that no R.P. begins with the starting values $a_{1}=1, a_{2} \geqslant 5$, while Section 6 deals with modular R.P.'s. In particular, such pairs are discussed in detail for the moduli 2 and 3. The section also contains a general existence question and two conjectures that arise from considerable computing.

Section 7 presents a theorem that determines the parity of the coefficients in the series appearing in identities 7 and 10. The paper closes with Section 8 in which partition interpretations of identities 5-10 are given that are analogous to those previously given for the Rogers-Ramanujan identities.

(b) In what follows, we will often use the $\left\{a_{i}\right\}$ sequence of an R.P. as the representative of the pair, since for a given $\left\{a_{i}\right\}$ sequence the corresponding $\left\{b_{j}\right\}$ sequence is uniquely determined. We will also classify the R.P.'s by their $\left\{a_{i}\right\}$ sequences, placing two such sequences in the same class if they have the same first term. Further, we will linearly order the sequences in each class by listing $\left\{a_{i}\right\}$ before $\left\{a_{i}^{\prime}\right\}$ if $a_{r}<a_{r}^{\prime}$ at the first term where the two sequences disagree. When this is done with the known pairs, we find that there are seven with $a_{1}=1$, five with $a_{1}=2$, and two in each class with $a_{1} \geqslant 3$.

These classes are represented diagrammatically in the form of row-indented trees Figures 1-3 below. (The first sequence in each class will clearly be 1 . in Table 1.) In each of these trees (where the consecutive terms in the given $\left\{a_{i}\right\}$ sequence are the nodes) every row past the first is considered to be connected by its first term to the nearest number in the preceding column in some row above. The rows are connected in no other way.

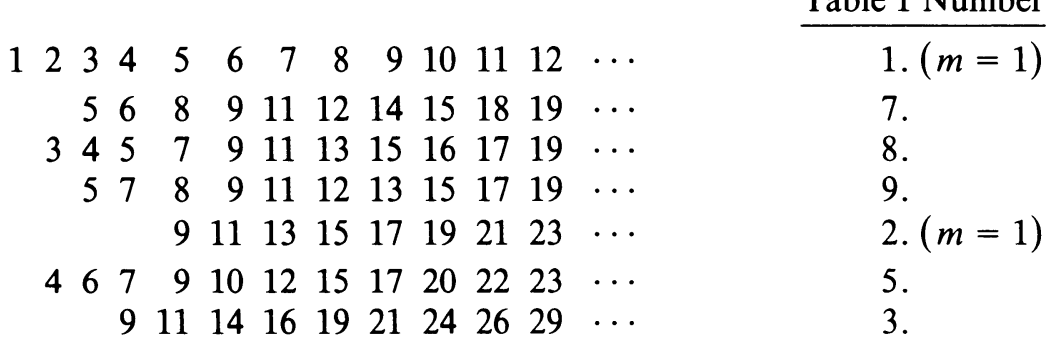

FIGURE 1. Tree for the first class

$\begin{array}{rrrrrrrrrrrrrr}2 & 3 & 4 & 5 & 6 & 7 & 8 & 9 & 10 & 11 & 12 & 13 & \cdots & 1 .(m=2) \\ & & & & & 13 & 14 & 15 & 16 & 17 & 18 & \cdots & 10 . \\ & & & 11 & 12 & 13 & 14 & 18 & 19 & 20 & 21 & \cdots & 6 . \\ & 5 & 7 & 9 & 11 & 13 & 15 & 17 & 19 & 21 & 23 & \cdots & 2 .(m=2) \\ & 7 & 8 & 12 & 13 & 17 & 18 & 22 & 23 & 27 & 28 & \cdots & 4 .\end{array}$

Figure 2. Tree for the second class

$$
\begin{array}{rrrrr}
m m+1 m+2 \cdots & 2 m-12 m 2 m+12 m+2 \ldots & 1 .(m \geqslant 3) \\
2 m+1 & 2 m+32 m+5 \cdots & 2 .(m \geqslant 3)
\end{array}
$$

FIGURE 3. Tree for the other classes

The question whether or not there are any other R.P.'s can now be expressed in terms of these trees by asking whether there is any other $\left\{a_{i}\right\}$ in an R.P. which 
branches off of one of the known sequences at some point, i.e., it agrees with this $\left\{a_{i}\right\}$ up to some term, but thereafter proceeds along a different sequence.

A nearly complete solution to this question will be published elsewhere by the first author who has shown in [4] that the only R.P.'s are those listed in Table 1, except possibly for pairs that might branch off of sequence 1 . in Table 1 for $m \leqslant 4$. Even in this case, considerable computing suggest there is no other R.P.

It should be noted that Lehmer [10] and Alder [2] have previously shown that certain pairs do not exist, where the exponents of the R.H.S. of (1) are generated by certain quadratic polynomials in $j$.

Finally, Acreman in his 1983 thesis [1] studied the coefficients on the two sides of (1) using asymptotic methods, an approach very different from our own. Using Hirschhorn's methods with the identities in [13] he obtained the R.P.'s 7. and 8., not knowing we had published these a year earlier in the Notices Amer. Math. Soc.

2. Finite Ramanujan Pairs. In what follows we will use the familiar abbreviation $(x)_{j}$ for the product $(1-x)\left(1-x^{2}\right) \cdots\left(1-x^{j}\right)$.

Definition 2.1. A pair of increasing sequences of positive integers $\left\{a_{i}\right\}_{i=1}^{m},\left\{b_{j}\right\}_{j=1}^{n}$ is called a finite Ramanujan Pair if the following equation holds:

$$
\prod_{i=1}^{m} \frac{1}{1-x^{a_{i}}}=1+\sum_{j=1}^{n} \frac{x^{b_{j}}}{(x)_{j}} .
$$

It is clear that not just one of the sequences $\left\{a_{i}\right\},\left\{b_{j}\right\}$ can be finite, for then $x=1$ would be a pole on one side of (2), but not on the other side.

In our later discussion, it will be important to know what all the finite R.P.'s are. These we determine in the next theorem.

THEOREM 2.2. The sequences $\left\{a_{i}\right\}_{i=1}^{m},\left\{b_{j}\right\}_{j=1}^{n}$ are a finite Ramanujan Pair $\Leftrightarrow m=$ $n$ and $a_{i}=b_{i}=i$ for each $i, 1 \leqslant i \leqslant n$.

Proof. $(\Leftarrow)$ This equation, which goes back to Euler [6], is readily established by induction on $n$.

$(\Rightarrow)$ When the fractions on the R.H.S. of (2) are added, we obtain a fraction with the form $A(x) /\left[(1-x)^{n} B(x)\right]$, where $A(x), B(x) \in \mathbf{Z}[x]$ and $A(1) B(1) \neq 0$. Thus, on the R.H.S. there is a pole of order $n$ at $x=1$. This implies there are exactly $n$ factors $1-x^{a_{i}}$ in the denominator on the L.H.S. of (2), so $m=n$.

We next show that $a_{n} \leqslant n$; for, if $a_{n}$ exceeded $n$, then the L.H.S. would have poles at the primitive $a_{n}$ th roots of unity, while the R.H.S. would only have poles at the primitive $k$ th roots of unity, with $k \leqslant n$. Thus, since $\left\{a_{i}\right\}$ is an increasing sequence, it follows that $a_{i}=i$ for each $i, 1 \leqslant i \leqslant n$. That $b_{i}=a_{i}$ follows by successively multiplying $(2)$ through by $(1-x),\left(1-x^{2}\right), \ldots,\left(1-x^{n}\right)$.

This theorem asserts that if we have any pair of finite sequences $\left\{a_{i}\right\}_{i=1}^{m},\left\{b_{j}\right\}_{j=1}^{n}$ other than those mentioned above, the infinite series on the two sides of (2) cannot agree to infinity, but will disagree at some first term whose degree we will call the discrepancy degree $d=d(m, n)$. We will also refer to the highest degree assigned in either sequence at this particular stage as the assignment degree $t$, i.e., $t=$ $\max \left\{a_{m}, b_{n}\right\}$.

Example 1. Let $\left\{a_{i}\right\}_{i=1}^{3}=\{2,3,5\}$ and $\left\{b_{j}\right\}_{j=1}^{2}=\{2,5\}$. Then

$$
\frac{1}{\left(1-x^{2}\right)\left(1-x^{3}\right)\left(1-x^{5}\right)}=1+x^{2}+x^{3}+x^{4}+2 x^{5}+2 x^{6}+2 x^{7}+\cdots,
$$


while

$$
1+\frac{x^{2}}{1-x}+\frac{x^{5}}{(1-x)\left(1-x^{2}\right)}=1+x^{2}+x^{3}+x^{4}+2 x^{5}+2 x^{6}+3 x^{7}+\cdots
$$

The coefficients in the two series first disagree at the 7th degree term, so $d=d(3,2)$ $=7$, while the highest degree assigned in the sequences so far is $t=a_{3}=b_{2}=5$.

3. The Search Method. We begin the search for a new R.P. by choosing values for $a_{1}, \ldots, a_{m}$, based on the trees in Figures $1-3$. The expansion of the product with these starting values gives the series

$$
\prod_{i=1}^{m} 1 /\left(1-x^{a_{i}}\right)=\sum_{k=0}^{\infty} \alpha_{k}^{(m)} x^{k}
$$

which forces the starting values for $b_{1}, \ldots, b_{n}, b_{n} \leqslant a_{m}$, in (1), so that the series

$$
1+\sum_{j=1}^{n} x^{b_{j}} /(x)_{j}=\sum_{k=0}^{\infty} \beta_{k}^{(n)} x^{k}
$$

agrees with the series in (3) up to degree $a_{m}$. (Many choices for $a_{1}, \ldots, a_{m}$ are, of course, impossible, because no corresponding $b$ 's exist for which the two series do agree up to degree $a_{m}$.)

If the initial $a_{i}$ choices are those for a finite R.P. (see Theorem 2.2), then the two series already agree to infinity and we set $d=\infty$. In what follows, however, we will not deal with this case any further. For any other initial choice, the two series will disagree at some degree $d$. We then try to extend the initial sequences so as to get the series in (3) and (4) to agree further. To do this, we can only make one of the following possible new assignments: $a_{m+1}=b_{n+1}=t$ for some $t$, where $a_{m}<t<d$ (called an " $a$ - $b$-assignment"), $a_{m+1}=d$ if $\alpha_{d}^{(m)}-\beta_{d}^{(n)}=-1$ (an " $a$-assignment"), or $b_{n+1}=d$ if $\alpha_{d}^{(m)}-\beta_{d}^{(n)}=1$ (a " $b$-assignment"). (Note that these latter two cases do not occur if $\left|\alpha_{d}^{(m)}-\beta_{d}^{(n)}\right|>1$, since any new assignment of an $a$ or $b$ value can never increase the coefficient of a term where the assignment is made by more than 1.)

Following each of these possible assignments, there is a new discrepancy degree and finitely many new possible assignments. This process is continued until either all the alternatives lead to a dead end, or there is a continuation that survives to the end of a particular search, i.e., up to some prespecified degree. A branch comes to a dead end when the only remaining choice requires an assignment to be made at a degree where the difference of the coefficients at that degree exceeds one. It is clear from the preceding description that the structure of the search is a tree search, where the nodes are the actual assignments, with an infinite branch giving an R.P. For a tree with only finite branches, there is no R.P. beginning with the particular starting values.

Example 2. Let $a_{1}=1$ and $a_{2}=3$. Then $b_{1}=1$ and $b_{2}=3$ are forced. We then have the following sequence of coefficients in (3) and (4) (Figure 4):

\begin{tabular}{c|cccccc}
$k$ & 0 & 1 & 2 & 3 & 4 & 5 \\
\hline$\alpha_{k}^{(2)}$ & 1 & 1 & 1 & 2 & 2 & 2 \\
$\beta_{k}^{(2)}$ & 1 & 1 & 1 & 2 & 2 & 3
\end{tabular}

FIGURE 4

\begin{tabular}{c|llllll}
$k$ & 0 & 1 & 2 & 3 & 4 & 5 \\
\hline$\alpha_{k}^{(3)}$ & 1 & 1 & 1 & 2 & 3 & 3 \\
$\beta_{k}^{(3)}$ & 1 & 1 & 1 & 2 & 3 & 4
\end{tabular}

Figure 5 
Thus, the initial assignments are possible, since the two series agree up to the assignment degree $t=3$. The discrepancy degree at this point is $d=5$, so there are just two possible ways of extending the sequences: (i) the $a-b$-assignment $a_{3}=b_{3}=$ 4; (ii) the $a$-assignment $a_{3}=5$.

If we choose (i), we obtain the coefficients in Figure 5 above. (Note that none of the coefficients of the terms up to and including the assignment degree are changed by later assignments.) Here $t=4$, while $d$ is still 5 . Thus, the next assignment $a_{4}=5$ is forced, giving the new coefficients (Figure 6):

\begin{tabular}{l|llllllll}
$k$ & 0 & 1 & 2 & 3 & 4 & 5 & 6 & 7 \\
\hline$\alpha_{k}^{(4)}$ & 1 & 1 & 1 & 2 & 3 & 4 & 5 & 6 \\
$\beta_{k}^{(3)}$ & 1 & 1 & 1 & 2 & 3 & 4 & 5 & 7
\end{tabular}

FIGURE 6

\begin{tabular}{c|ccc}
$k$ & $\ldots \ldots$ & 7 & 8 \\
\hline$\alpha_{k}^{(5)}$ & $\ldots \ldots$ & 7 & 9 \\
$\beta_{k}^{(3)}$ & $\ldots \ldots$ & 7 & 8
\end{tabular}

FIGURE 7

Once again there are two possible assignments: (i) $a_{5}=b_{4}=6$; (ii) $a_{5}=7$. We will end this example by following (ii) two more steps.

With $a_{5}=7$, we obtain the series in Figure 7 (only the necessary coefficients are shown), so the next assignment $b_{4}=8$ is forced. We then obtain Figure 8 , where we list only the coefficients from $t=8$ to $d=15$ :

\begin{tabular}{l|rrrrrrrr}
$k$ & 8 & 9 & 10 & 11 & 12 & 13 & 14 & 15 \\
\hline$\alpha_{k}^{(5)}$ & 9 & 11 & 14 & 17 & 21 & 25 & 30 & 36 \\
$\beta_{k}^{(4)}$ & 9 & 11 & 14 & 17 & 21 & 25 & 30 & 35
\end{tabular}

\section{FIGURE 8}

Here we unexpectedly have six equal coefficients at degrees above the assignment degree but less than the discrepancy degree 15 . The search therefore breaks into seven possible cases at this point.

Example 3. To illustrate a dead end, we return to Example 1 with $a_{1}=2, a_{2}=3$, $a_{3}=5, b_{1}=2, b_{2}=5$ and the coefficients given in Figure 9:

\begin{tabular}{c|llllllll}
$k$ & 0 & 1 & 2 & 3 & 4 & 5 & 6 & 7 \\
\hline$\alpha_{k}^{(3)}$ & 1 & 0 & 1 & 1 & 1 & 2 & 2 & 2 \\
$\beta_{k}^{(2)}$ & 1 & 0 & 1 & 1 & 1 & 2 & 2 & 3
\end{tabular}

FIGURE 9

\begin{tabular}{c|lll}
$k$ & 5 & 6 & 7 \\
\hline$\alpha_{k}^{(4)}$ & 2 & 3 & 2 \\
$\beta_{k}^{(3)}$ & 2 & 3 & 4
\end{tabular}

FiguRE 10

Here there are the two choices: (i) $a_{4}=b_{3}=6$; (ii) $a_{4}=7$. The first gives Figure 10 above. Since $t=6$ and $d=7$ and the difference in the coefficients at degrees 7 is 2 , we have reached a dead end and no further assignment can be made.

It should be clear from the preceding example that the actual tree of assignments that extend a given starting pair consists of the degrees at which the assignments are made. Trees are presented in the row-indented form of Figures 1-3, where dashes connect $a$ - or $a b$-assignments and an asterisk follows a $b$-assignment. This is illustrated by the complete search tree (Figure 11) for the initial sequence $a_{1}=1$, $a_{2}=6$. The finiteness of this tree proves that there is no R.P. beginning in this way. 
The tree in Figure 11 shows that all branches end (arrive at a contradiction) by degree 40 . If one were therefore to follow the proof by actually computing the series in (3) and (4) along all the branches, he would only have to deal with the terms of the series $\left(\bmod x^{40}\right)$. In general, for any initial sequence, one does not know in advance how long the branches of the search tree will be; however, a great many starting sequences can be eliminated by computing $\left(\bmod x^{150}\right)$ or $\left(\bmod x^{200}\right)$, as we have found by experience. Any short starting sequence that can be extended so that the series in (3) and (4) agree out this far is a very good candidate for a true R.P.

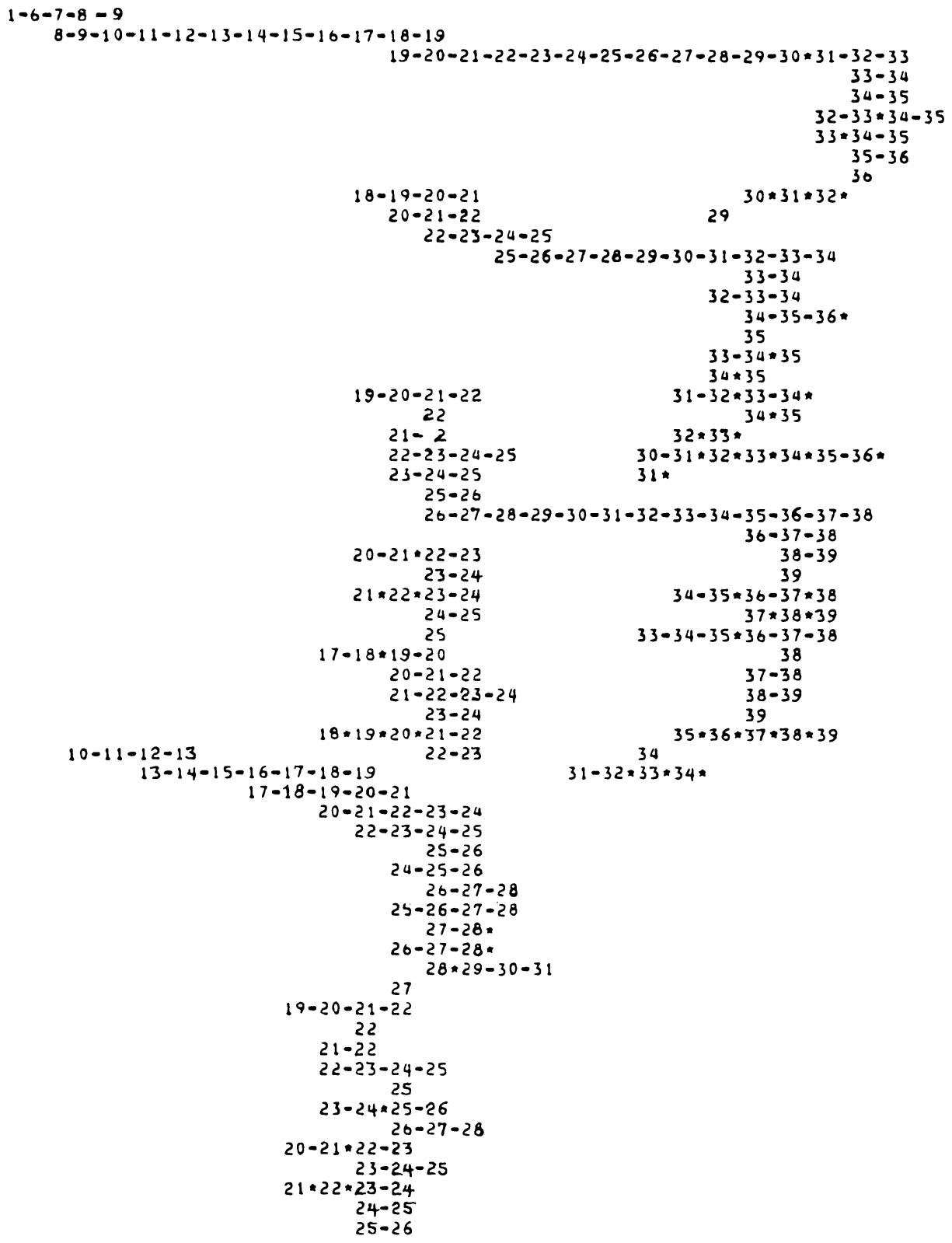

FIGURE 11. Complete Search Tree for $a_{1}=1, a_{2}=6$ 


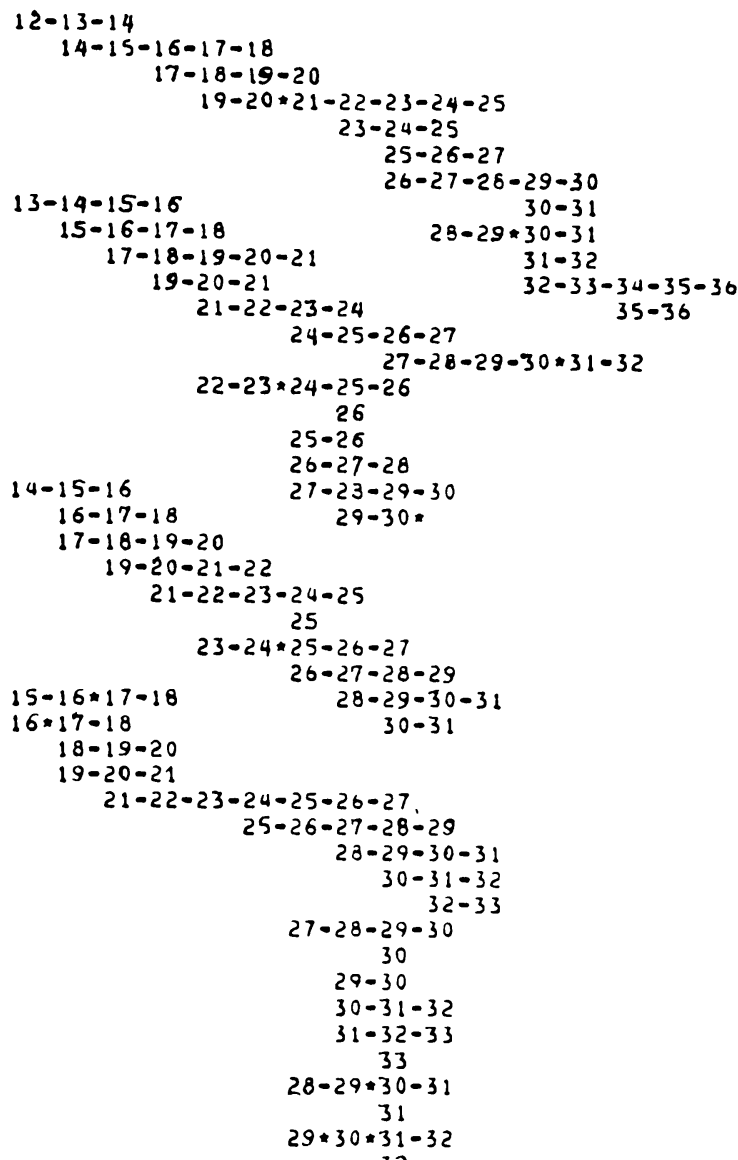

Figure 11 (Continued)

The four new R.P.'s were discovered by computing $\left(\bmod x^{150}\right)$ (this power is an input parameter to the program) and finding agreement in the two series up to this point. Further computation showed that the series agreed up to degree 200 . We concluded then that it was highly likely that we were dealing with the beginning of a true R.P. When the first differences of the $a_{i}$ and $b_{j}$ sequences were computed, in order to try to recognize the sequences, it was apparent that the sequence $\left\{a_{i}\right\}$ was periodic $(\bmod 20)$. The conjectured form of the four identities could then be readily written down. Shortly thereafter, it was discovered that these conjectured identities were derivable from four identities already published in Slater and so were therefore true.

One interesting "end effect" for a true R.P., when the calculations are made only $\left(\bmod x^{N}\right)$ for some $N$, is that bogus candidates sometimes appear that agree with the true R.P. up to just below degree $N$ and then deviate a little from it. The bogus pair is due to a "fluttering" end effect which can easily be eliminated by raising the value of $N$ automatically in the program by, say, 50 or so, and then recomputing the series $\left(\bmod x^{N}\right)$ for the larger value of $N$. 
4. The Search Programs. A computer program named RAMAN was written to explore the search tree described in the previous section up to a prespecified maximum degree $N$. Typically $N$ was 200 , although it could be as large as 1500 . Actually, three versions of RAMAN were developed. The first, RAMAN1, is an interactive program which allows the user to choose the assignments. The other two programs search the tree automatically. They differ in that RAMAN2 searches each branch to its absolute dead end, or else to degree $N$, while RAMAN3 uses exclusion criteria to detect and abort a "hopeless" branch before it is actually searched to its end.

The decision at each particular node of the search tree as to what the next assignment should be is based on a comparison of the coefficients on the right sides of (3) and (4). These coefficients grow quickly in size. So as to avoid unnecessary multiple-precision calculations, the coefficients were computed $\left(\bmod 2^{16}\right)$ on our 16 bit machine. This modular reduction was sufficient for our purposes, since only the difference of the coefficients $\alpha_{k}^{(m)}-\beta_{k}^{(n)}$ is needed each time an assignment is made. Of course, this means we are actually searching for R.P.'s in which the coefficients of the series are taken $\left(\bmod 2^{16}\right)$. Such modular R.P.'s are discussed in Section 6.

All three versions of RAMAN use the same procedures to calculate the series in (3) and (4) modulo $x^{N+1}$. The method for computing the coefficients on the right side of $(3)$ is based on the following inductive formula.

Proposition 4.1. Suppose $a_{m+1}=t$. Then,

$$
\begin{array}{lll}
\text { (a) } \alpha_{k}^{(m+1)}=\alpha_{k}^{(m)}, & 0 \leqslant k<t, \\
\text { (b) } \alpha_{k}^{(m+1)}=\alpha_{k}^{(m)}+\alpha_{k-t}^{(m+1)}, & & k \geqslant t .
\end{array}
$$

In computing the right side of (4) we need the additional series

$$
\prod_{j=1}^{n}\left(1-x^{j}\right)^{-1}=\sum_{k=0}^{\infty} \rho_{k}^{(n)} x^{k} .
$$

The coefficients $\rho_{k}^{(n)}$ are easily obtained by Proposition 4.1. We use them to compute the coefficients $\beta_{k}^{(n)}$ in (4) as follows:

Proposition 4.2. Suppose $b_{n+1}=t$. Then,

$$
\begin{aligned}
& \text { (a) } \beta_{k}^{(n+1)}=\beta_{k}^{(n)}, \quad 0 \leqslant k<t \text {, } \\
& \text { (b) } \beta_{k}^{(n+1)}=\beta_{k}^{(n)}+\rho_{k-t}^{(n+1)}, \quad k \geqslant t \text {. }
\end{aligned}
$$

The proofs of Propositions 4.1 and 4.2 involve straightforward series manipulation, and are left to the reader.

Now, when we reach a dead end in the search, we must be able to "back up" the series coefficients, in order to try other paths of the search tree. To obtain $\alpha_{k}^{(m-1)}$ from $\alpha_{k}^{(m)}$, we simply solve for $\alpha_{k}^{(m-1)}$ in (5), where $m$ is replaced by $m-1$.

Proposition 4.3. Let $m \geqslant 2$ and $a_{m}=t$. Then,

$$
\begin{array}{lll}
\text { (a) } & \alpha_{k}^{(m-1)}=\alpha_{k}^{(m)}, & 0 \leqslant k<t, \\
\text { (b) } \alpha_{k}^{(m-1)}=\alpha_{k}^{(m)}-\alpha_{k-t}^{(m)}, & k \geqslant t .
\end{array}
$$


Similarly, from (7) we derive

Proposition 4.4. Let $n \geqslant 2$ and $b_{n}=t$. Then,

$$
\begin{aligned}
& \text { (a) } \beta_{k}^{(n-1)}=\beta_{k}^{(n)}, \quad 0 \leqslant k<t \text {, } \\
& \text { (b) } \beta_{k}^{(n-1)}=\beta_{k}^{(n)}-\rho_{k-t}^{(n)}, \quad k \geqslant t \text {. }
\end{aligned}
$$

Corresponding to the current values of $m, n$, and the sequences $\left\{a_{i}\right\}_{i=1}^{m},\left\{b_{j}\right\}_{j=1}^{n}$, all three versions of RAMAN store the current values of the series coefficients $\alpha_{k}^{(m)}$, $\beta_{k}^{(n)}$, and $\rho_{k}^{(n)}$ in the arrays $\alpha=\left\{\alpha_{k}\right\}_{k=0}^{N}, \beta=\left\{\beta_{k}\right\}_{k=0}^{N}$, and $\rho=\left\{\rho_{k}\right\}_{k=0}^{N}$, respectively. For the next assignment $a_{m}=t$, RAMAN updates the array $\alpha$ by implementing (5), which involves a successive shifting and adding of the array $\alpha$ to itself up to index $N$. When backing up, RAMAN uses (8) and (9). Note that the iteration specified in (8b) and (9b) must be performed in decreasing order from $k=N$ down to $k=t$ to prevent "writing over" entries prematurely.

We now discuss the three versions of RAMAN. The interactive program RAMAN1 computes the coefficients in (3) and (4) by Propositions 4.1 through 4.4, and displays these to the user. The coefficients $\alpha_{k}$ and $\beta_{k}$ appear in two rows on a terminal screen inside a "window" centered about the first discrepancy in coefficients. (The window size is specified at the beginning of the run and is usually taken to be 10.) At this stage the computer asks the user to make one of the following decisions:

1. Continue the search by choosing a new assignment degree.

2. Back up the series to a previous degree $d$, i.e., eliminate all assignments past degree $d$ and recompute the coefficients in $\alpha$ and $\beta$ accordingly.

3. Print out a copy of $\alpha$ and $\beta$.

4. Stop.

Incidentally, RAMAN1 automatically performs forced assignments up to the assignment degree that was input. If it detects an unresolvable discrepancy before it reaches the assignment degree, then it outputs an error message.

The second program RAMAN2 did the tree search automatically and at high speed. In addition to the arrays $\boldsymbol{\alpha}, \boldsymbol{\beta}$, and $\boldsymbol{\rho}$, this program uses two "history" arrays $\left\{d_{i}\right\}_{i=1}^{r}$ and $\left\{f_{i}\right\}_{i=1}^{r}$. Here $d_{i}$ is the $i$ th assignment degree and $f_{i}$ is the type of assignment made at degree $d$, according to the rule:

$$
f_{i}=\left\{\begin{array}{cl}
-1, & \text { for an } a \text {-assignment } \\
0, & \text { for an } a b \text {-assignment } \\
1, & \text { for a } b \text {-assignment }
\end{array}\right.
$$

We now give in some detail the search method employed by RAMAN2.

Algorithm 4.5. Given maximum degree $N$ and initial sequences $\left\{a_{i}\right\}_{i=1}^{m_{0}},\left\{b_{j}\right\}_{j=1}^{n_{0}}$. Find all pairs $\left\{a_{i}\right\}_{i=1}^{m},\left\{b_{j}\right\}_{j=1}^{n}$ which satisfy (1) modulo $x^{N+1}$.

\section{I (Initialize variables)}

1. Input $N,\left\{a_{i}\right\}_{i=1}^{m_{0}},\left\{b_{j}\right\}_{j=1}^{n_{0}}$.

2. Put $t=\max \left\{a_{m_{0}}, b_{n_{0}}\right\}$. (Here we assume that the initial sequences satisfy (1) $\left(\bmod x^{t+1}\right)$.)

3. Set $m=m_{0}, n=n_{0}$, and $r=0$. 
4. Compute the coefficient series $\alpha, \boldsymbol{\beta}$, and $\rho$ corresponding to $\left\{a_{i}\right\}_{i=1}^{m}$ and $\left\{b_{j}\right\}_{j=1}^{n}$ by repeated use of (5) and (7).

II (Search Module)

1. If $t \geqslant N$, then a solution has been found up to degree $N$. In this case, output the pair $\left\{a_{i}\right\}_{i=1}^{m},\left\{b_{j}\right\}_{j=1}^{n}$, then go to IV.

2. Increment $t$ by 1 .

3. Set $\delta=\alpha_{t}-\beta_{t}$.

4. (Check for a dead end.) If $|\delta| \geqslant 2$, go to IV.

5. (Update the history arrays.) Increment $r$ by 1 . Set $d_{r}=t, f_{r}=\delta$.

III (Assignment Module)

1 . If $\delta \leqslant 0$, increment $m$ by 1 and use (5) to update $\alpha$.

2. If $\delta>0$, increment $n$ by 1 ; use Proposition 4.1 to update $\rho$ and (7) to update $\boldsymbol{\beta}$.

3. Go to II.

IV (Reverse: Backtrack in the Tree Search)

1. If $r=0$, stop; all paths of the finite search tree have been examined.

2. Set $t=d_{r}, \delta=f_{r}$.

3. If $\delta \leqslant 0$, decrement $m$ by 1 and use (8) to back up $\alpha$.

4. If $\delta>0$, decrement $n$ by 1; use Proposition 4.3 to back up $\rho$ and (9) to back up $\beta$.

5. Decrement $r$ by 1 .

6. If $\delta=0$, go to II. Otherwise go to IV.

In implementing this algorithm, an input parameter $\Delta N$ is specified along with the maximum degree $N$, so that RAMAN2 outputs all sequences $\left\{a_{i}\right\}_{i=1}^{m},\left\{b_{j}\right\}_{j=1}^{n}$ which satisfy (1) modulo $x^{N+1}$ and which can be extended to sequences which satisfy (1) up to the higher degree $N+\Delta N$. Typically, $\Delta N$ was about 50 . This technique eliminated the "fluttering" end effects mentioned at the end of Section 3. In addition to any pairs that might have been found in the search, the program outputs final statistics on the number of assignments, dead ends, vertices, and paths, along with the maximum degree reached. These give a rough idea of the structure and complexity of the search tree. For many initial starts, RAMAN2 found that every branch of the search tree dead-ended before degree $N$. In such cases, a simple tree structure or a repeating pattern in the output of the search program, suggests theoretical nonexistence proofs. An example of this is Theorem 5.3 in the next section, where values of $s \geqslant 7$ gave almost identical search trees.

The other automatic search program, RAMAN3, is an "early abort" version of RAMAN2, which uses exclusion criteria to avoid going all the way to the end of a hopeless branch.

Example 4. Let $a_{1}=10, \ldots, a_{10}=19$ and $b_{1}=10, b_{2}=22$. Then the $\alpha_{k}^{(10)}, \beta_{k}^{(2)}$ coeffients of the series in (3) and (4) are

\begin{tabular}{c|rrrrrrrrrrr}
$k$ & 20 & 21 & 22 & 23 & 24 & 25 & 26 & 27 & 28 & 29 & 30 \\
\hline$\alpha_{k}=\alpha_{k}^{(10)}$ & 1 & 1 & 2 & 2 & 3 & 3 & 4 & 4 & 5 & 5 & 5 \\
$\beta_{k}=\beta_{k}^{(2)}$ & 1 & 1 & 2 & 2 & 3 & 3 & 4 & 4 & 5 & 5 & 6
\end{tabular}


Here the assignment degree is 22 , and the discrepancy degree is 30 . By the search method described in Section 3, we have 8 possible choices at this point: $7 a b$-assignments at degrees $t=23, \ldots, 29$, or else the single $a$-assignment at degree 30 . Note, however, that $\sum \alpha_{k} x^{k}$ begins $1+x^{10}+\cdots$. By (5), assigning $a_{11}$ to be any value from 23 to 29 cannot change the coefficient $\alpha_{30}=5$. On the other hand, an assignment of $b_{3}$ at any degree 23 to 29 will increase $\beta_{30}=6$ by at least 1 . Thus, each of the $7 a b$-assignments will dead-end by degree 30 .

RAMAN3 was sophisticated enough to recognize these "doomed" branches. For Example 4, it would only assign $a_{11}=30$, whereas RAMAN2 would examine all 8 branches. Of course, this required a more complicated code, and therefore was only of value for long-running cases. RAMAN3 also provided a check on RAMAN2 since in all cases checked the results of these independent programs agreed.

Finally, a fourth program TREE was written which uses special output of RAMAN2 to produce a visual display of the search tree in the indented format of Figure 11.

5. A Nonexistence Theorem. An examination of Figure 1 shows that there are known R.P.'s starting with $a_{1}=1$ and $a_{2}=2,3$, and 4 , but none for $a_{2}>4$. By computing the trees for some of these higher values of $a_{2}$, it became clear that their structure was sufficiently simple and similar that a general theoretical nonexistence proof could be made.

Before undertaking this proof, we first establish some useful lemmas that relate to the equation

$$
\prod_{i=n}^{\infty}\left(1-x^{a_{i}}\right)^{-1}=1+\sum_{k=1}^{\infty} \alpha_{k} x^{k}
$$

where $a_{n}<a_{n+1}<\cdots$ is a sequence of positive integers. The proofs of the following lemmas depend on the fact that $\alpha_{k}=\alpha_{k}(n)$ is the number of partitions of $k$ into the parts $a_{n}, a_{n+1}, \ldots$

LeMMA 5.1. For each $k, 1 \leqslant k \leqslant 2 a_{n}-1, \alpha_{k}=0$ or 1 .

Proof. The smallest integer having a partition into at least two parts is clearly $2 a_{n}=a_{n}+a_{n}$. Hence, there is at most one partition for each $k$ in the interval $\left[1,2 a_{n}-1\right]$.

Lemma 5.2. Suppose $a_{n}$ is even and let $a_{m}, m>n$, be the first odd term in the sequence $\left\{a_{i}\right\}$. Then $\alpha_{k}=0$ or 1 for each odd $k$, where $1 \leqslant k<a_{m}+a_{n}$.

Proof. The smallest odd number having a partition into at least two parts is clearly $k=a_{m}+a_{n}$.

THEOREM 5.3. There is no Ramanujan Pair with $a_{1}=1, a_{2}=s, s \geqslant 5$.

Proof. A comparison of the two sides of the equation

$$
\frac{1}{(1-x)\left(1-x^{s}\right)} \prod_{i=3}^{\infty}\left(1-x^{a_{i}}\right)^{-1}=1+\sum_{j=1}^{\infty} \frac{x^{b_{j}}}{(1-x)\left(1-x^{2}\right) \cdots\left(1-x^{j}\right)}
$$

yields successively that $b_{1}=1, b_{2}=s$, and $b_{3} \geqslant s+1$. We now consider two cases, depending on the parity of $s$. 
Case 1. $s$ even. Put $s=2 r, r \geqslant 4$. (We will handle $s=6$ separately.) Multiplying (11) through by $(1-x)\left(1-x^{s}\right)$ we obtain the equation

$$
\begin{aligned}
\prod_{i=3}^{\infty}(1 & \left.-x^{a_{i}}\right)^{-1} \\
= & 1+x^{2 r+2}+x^{2 r+4}+\cdots+x^{4 r-2} \\
& +x^{b_{3}}\left(1+x^{2}+x^{3}+x^{4}+x^{5}+2 x^{6}+x^{7}+2 x^{8}+2 x^{9}+\cdots\right) \\
& -x^{b_{3}+2 r}\left(1+x^{2}+x^{3}+\cdots\right)+\cdots .
\end{aligned}
$$

Let $a_{m}$ be the first odd term in the sequence $a_{3}, a_{4}, \ldots$ (Not all the terms $a_{i}$, $i \geqslant 3$, can be even, for then the coefficients of the odd powers in the series expansion of the L.H.S. of (12) would all be 0.) We have $a_{m} \geqslant b_{3}$, since all the preceding powers of $x$ on the right of (12) are even. If $b_{3}$ is odd, then $a_{m}=b_{3}$ and we find that the coefficient of the term $x^{b_{3}+6}$ on the right of (12) is $\geqslant 2$. This contradicts Lemma 5.2 , since $b_{3}+6<a_{m}+a_{3}$. On the other hand, suppose $b_{3}$ is even. Then the coefficient of the term $x^{b_{3}+9}$ is $\geqslant 2$, again contradicting Lemma 5.2, since $b_{3}+9<$ $a_{m}+9 \leqslant a_{m}+a_{3}$. (Note that if $r=4$, the terms with negative coefficients on the R.H.S. of (12) do not affect the $x^{b_{3}+9}$ term.)

The remaining part of this case, $s=6$, is contained in the complete search tree-which is finite-that is given in Figure 11. For this proof to be convincing, it is necessary for the reader to start with $a_{1}=1$ and $a_{2}=6$ and trace through the alternatives detailed in the tree to check that it is complete and correct. We prefer this mode of proof to an unnecessarily complicated theoretical proof. The graphic proof is concise and easy to verify, especially with an interacting program.

Case 2. $s=2 r+1, r \geqslant 2$. Again, with $b_{1}=1, b_{2}=s$, and $b_{3} \geqslant s+1$, we multiply (11) through by $(1-x)\left(1-x^{2 r+1}\right)$, obtaining the congruence

$$
\begin{aligned}
\prod_{i=3}^{\infty}(1 & \left.-x^{a_{i}}\right)^{-1} \\
= & 1-x^{2 r+1}+\left(x^{2 r+1}-x^{4 r+2}\right)\left(1+x^{2}+x^{4}+\cdots+x^{2 r+4}\right) \\
& +\left(x^{b_{3}}-x^{2 r+1+b_{3}}\right)\left(1+x^{2}+x^{4}+\cdots\right)\left(1+x^{3}+x^{6}+\cdots\right) \\
\equiv & 1+\left(x^{2 r+3}+x^{2 r+5}+\cdots+x^{4 r+5}\right)+x^{b_{3}}\left(1+x^{2}+x^{3}+\cdots\right) \\
& -x^{4 r+2}-x^{4 r+4}-x^{2 r+1+b_{3}}-x^{2 r+3+b_{3}}+\cdots \quad\left(\bmod x^{2 r+6}\right) .
\end{aligned}
$$

The minimal choice, $b_{3}=2 r+2$, produces on the right the leading five terms $1+x^{2 r+2}+x^{2 r+3}+x^{2 r+4}+2 x^{2 r+5}$, which cannot be balanced by any choice of $a$ 's on the left because of the coefficient 2 . Since the assignment $b_{3}=2 r+3$ would also produce a coefficient 2 for $x^{2 r+3}$ on the right, we must have $b_{3} \geqslant 2 r+4$. But this forces the assignment $a_{3}=2 r+3$. Multiplying (11) through by $1-x^{a_{3}}$ gives

$$
\begin{gathered}
\prod_{i=4}^{\infty}\left(1-x^{a_{i}}\right)^{-1} \equiv \\
-x^{2 r+5}+x^{2 r+7}+\cdots+x^{4 r+5}+x^{b_{3}}\left(1+x^{2}+x^{3}+\cdots\right) \\
-x^{4 r+2}-x^{4 r+4}-x^{2 r+1+b_{3}}+\cdots \quad\left(\bmod x^{4 r+6}\right) .
\end{gathered}
$$

Now the minimal choices $b_{3}=2 r+4$ and $b_{3}=2 r+5$ again lead to a contradiction by the reasoning above, so we must have $b_{3} \geqslant 2 r+6$ and $a_{4}=2 r+5$. 
Continuing in this way, we finally reach the congruence

$$
\begin{aligned}
\prod_{i=r+3}^{\infty}\left(1-x^{a_{i}}\right)^{-1} \equiv & 1+x^{4 r+3}+x^{4 r+5}+x^{b_{3}}\left(1+x^{2}+x^{3}+\cdots\right) \\
& -x^{4 r+2}-x^{4 r+4}+\cdots\left(\bmod x^{4 r+6}\right)
\end{aligned}
$$

where $b_{3} \geqslant 4 r+2$, in which the term of smallest degree on the right of (14) is $-x^{4 r+2}$, which forces the assignment $b_{3}=4 r+2$. But then the coefficient of $x^{4 r+5}$ is 2 , a contradiction.

6. Modular Ramanujan Pairs. As mentioned in Section 4, in our search for R.P.'s, the coefficients $\alpha_{k}^{(m)}, \beta_{k}^{(n)}$ in (3) and (4) were all computed $\left(\bmod 2^{16}\right)$. This inspires the following

Definition 6.1. Given $M \geqslant 2$. Two finite or infinite increasing sequences** $\left\{a_{i}\right\}_{i=1}^{r}$, $\left\{b_{j}\right\}_{j=1}^{s}$ in $\mathbf{N}$ is called a Ramanujan Pair modulo $M$ if

$$
\prod_{i=1}^{r}\left(1-x^{a_{i}}\right)^{-1} \equiv 1+\sum_{j=1}^{s} x^{b_{j}} /(x)_{j} \quad(\bmod M),
$$

where the coefficients of the series on both sides of (15) are reduced modulo $M$.

Thus, RAMAN actually searches for R.P.'s $\left(\bmod 2^{16}\right)$. It is clear that if $\left\{a_{i}\right\},\left\{b_{j}\right\}$ is an R.P., then it will also be an R.P. $(\bmod M)$ for any $M \geqslant 2$. Consequently, the nonexistence of an R.P. $\left(\bmod 2^{16}\right)$ implies the nonexistence of the ordinary R.P., i.e., nonexistence arguments using RAMAN are valid. Conversely, RAMAN will certainly find the $\left(\bmod 2^{16}\right)$ "image" of a genuine R.P. Still, it is possible-although highly unlikely - that RAMAN could find the beginning of a modular R.P. $\left(\bmod 2^{16}\right)$ which is not the image of an ordinary R.P. This possibility raises the general question: For which $M \geqslant 2$ do there exist R.P.'s $(\bmod M)$ which are not ordinary R.P.'s? This question is answered affirmatively for $M=2$ in Theorem 6.2 and for $M=3$ in Theorem 6.3, following which, the case for $M \geqslant 4$ is discussed.

Let $\mathscr{S}$ be the set of all finite or infinite increasing sequences of positive integers. We now show that there exist infinitely many R.P.'s $(\bmod 2)$.

THEOREM 6.2. For any $\mathbf{a} \in \mathscr{S}$, there exists a unique sequence $\mathbf{b} \in \mathscr{S}$ such that $\mathbf{a}, \mathbf{b}$ is an R.P. $(\bmod 2)$. The map $\tau: \mathscr{S} \rightarrow \mathscr{S}$ given by $\tau(\mathbf{a})=\mathbf{b}$ is a bijection.

Proof. For $\mathbf{a}=\left\{a_{i}\right\}_{i=1}^{r} \in \mathscr{S}(r \in \mathbf{N}$ or $r=\infty)$, let

$$
\alpha(x)=\sum_{k=0}^{\infty} \alpha_{k} x^{k} \equiv \prod_{i=1}^{r}\left(1-x^{a_{i}}\right)^{-1}(\bmod 2),
$$

where $\alpha_{k} \in\{0,1\}, k \geqslant 0$. We describe an inductive algorithm for obtaining $\mathbf{b}=$ $\left\{b_{j}\right\}_{j=1}^{s}$ for some $s$ (possibly $\left.s=\infty\right)$ such that a, b is an R.P. ( $\left.\bmod 2\right)$. First set $b_{1}=a_{1}$, so $\alpha(x) \equiv 1+x^{b_{1}} /(1-x)\left(\bmod x^{b_{1}+1}, 2\right)$. Then assume inductively that $b_{1}<b_{2}<\cdots<b_{k}$ have been obtained so the congruence

$$
\alpha(x) \equiv 1+\sum_{j=1}^{k} x^{b_{k}} /(x)_{j}(\bmod 2)
$$

** Here $r$ and $s$ can be positive integers or $\infty$. 
holds modulo $x^{b_{k}+1}$. If, in fact, (17) is true, then take $s=k$ and $\mathbf{b}=\left\{b_{1}, \ldots, b_{s}\right\}$. Otherwise, take $b_{k+1}$ to be the first power for which the coefficients in (17) disagree $(\bmod 2)$. Clearly, $b_{k+1}>b_{k}$ and

$$
\alpha(x) \equiv 1+\sum_{j=1}^{k+1} x^{b_{j}} /(x)_{j} \quad\left(\bmod x^{b_{k+1}+1}, 2\right) .
$$

In this way we generate $\mathbf{b}$. If the process continues indefinitely, then $s=\infty$.

It is easy to show that the b sequence of an R.P. a, b modulo $M$ is uniquely determined. Conversely, $\mathbf{a}$ is also uniquely determined from $\mathbf{b}$, so the map $\tau: \mathbf{a} \rightarrow \mathbf{b}$ is $1-1$. To see that $\tau$ is onto, observe that given $\mathbf{b} \in \mathscr{S}$, we can use a procedure similar to the above algorithm to generate the corresponding a.

Theorem 6.2 shows that modulo 2 , $\mathbf{a}$ or $\mathbf{b}$ can be any finite sequence. Considering the importance of finite R.P.'s which was seen earlier, we ask:

Do there exist finite sequences $\left\{a_{i}\right\}_{i=1}^{m}$ and $\left\{b_{j}\right\}_{j=1}^{n}$, other than those of Theorem 2.2 , which satisfy (15) for some $M \geqslant 2$ ?

The search method of Section 3 can be modified to search for R.P.'s $(\bmod M)$. Suppose that $\left\{a_{i}\right\}_{i=1}^{m},\left\{b_{j}\right\}_{j=1}^{n}$ satisfy congruence (15) modulo $x^{a_{m}+1}$. (Here assume $b_{n} \leqslant a_{m}$.) Use (3) and (4) to obtain the coefficients $\alpha_{k}^{(m)}, \beta_{k}^{(n)}$. Then we define $d_{M}=d_{M}(m, n)$, the discrepancy degree $(\bmod M)$, to be the first index $k$ for which $\alpha_{k}^{(m)} \not \equiv \beta_{k}^{(n)}(\bmod M)$. As in Section 3, we can make the following assignments: $a_{m+1}=b_{n+1}=t$ for some $t, a_{m}<t<d_{M} ; a_{m+1}=d_{M}$ if $\alpha_{d_{M}}^{(m)}-\beta_{d_{M}}^{(n)} \equiv-1$ $(\bmod M) ; b_{n+1}=d_{M}$ if $\alpha_{d_{M}}^{(m)}-\beta_{d_{M}}^{(n)} \equiv 1(\bmod M)$. This process generates the search tree modulo $M$.

Now suppose $M=3$. For any integer $k \geqslant 0$, certainly $\alpha_{k}^{(m)}-\beta_{k}^{(n)} \equiv-1,0$, or 1 $(\bmod 3)$. Thus, since the discrepancy is never 2 , the search tree $(\bmod 3)$, once started, can never dead-end! This proves

THEOREM 6.3. Let $\left\{a_{i}\right\}_{i=1}^{m},\left\{b_{j}\right\}_{j=1}^{n}$ be any pair of finite sequences in $\mathscr{S}$ which satisfy (15) with $M=3$, up to degree $\max \left\{a_{m}, b_{n}\right\}$. Then $\left\{a_{i}\right\}_{i=1}^{m},\left\{b_{j}\right\}_{j=1}^{n}$ can be completed to an R.P. $(\bmod 3)$.

Example 5. In Example 3 in Section 3, the coefficients $\alpha_{k}^{(4)}$ and $\beta_{k}^{(3)}, 0 \leqslant k \leqslant 7$, are shown in Figures 9 and 10. Since $\alpha_{7}^{(4)}-\beta_{7}^{(3)}=2-4=-2$, the search method of Section 3 dead-ends at this point. Modulo 2, however, these coefficients agree at degree 7 , while modulo 3 , we are forced to assign $b_{4}=7$.

We now state two conjectures, relating to the modular R.P.'s for $M \geqslant 4$, which have emerged from the considerable computing we have done.

Conjecture 1. There exists a $B \in \mathbf{N}$ such that for every pair $\left\{a_{i}\right\}_{i=1}^{m},\left\{b_{j}\right\}_{j=1}^{n}$ of increasing sequences in $\mathbf{N}$ for which $\alpha_{k}^{(m)}=\beta_{k}^{(n)}, 0 \leqslant k \leqslant \max \left\{a_{m}, b_{n}\right\}$, we have $\left|\alpha_{d}^{(m)}-\beta_{d}^{(n)}\right| \leqslant B$, where $d=d(m, n)$ is the discrepancy degree.

The next example shows that $B \geqslant 5$.

Example 6. Let $\left\{a_{i}\right\}_{i=1}^{22}=\{6,7, \ldots, 34,35,41,42\}$ and $\left\{b_{j}\right\}_{j=1}^{9}=\{6,12,18,24$, $30,38,39,40,41\}$. Then the coefficients $\alpha_{k}^{(22)}$ and $\beta_{k}^{(9)}$ agree up to degree 42 , while $\alpha_{43}^{(22)}-\beta_{43}^{(9)}=245-250=-5$. Here the search for an ordinary R.P. ends at degree 43. But for $M=4,5$, and 6 , note that the search for an R.P. $(\bmod M)$ has "new life" at this point. The reprieve is only temporary, however, as the search trees modulo $M$ dead-end by degrees 74,47 , and 44 for $M=4,5$, and 6, respectively. 
As a second example, we consider the search tree beginning $a_{1}=1, a_{2}=6$. This tree, displayed in Figure 11, has 465 vertices, 136 paths, and the highest degree reached is 39. Modulo 5, the search tree extends to degree 44, with 11 more vertices and 2 more paths, while the tree diagram $(\bmod 4)$ is considerably more elaborate: 1381 vertices, 357 paths, and maximum degree 93. Thus, the search trees modulo $M=4$ and 5 that begin with $a_{1}=1, a_{2}=6$, although more complicated than Figure 11, are nonetheless finite. We are led to make

Conjecture 2 . For $M \geqslant 4$, the only R.P.'s $(\bmod M)$ are those derived from R.P.'s by considering them $(\bmod M)$.

Observe that Conjecture 1 implies Conjecture 2 for $M \geqslant B+2$.

7. A Parity Theorem. Since the coefficients of the series we have dealt with in this work were computed $\left(\bmod 2^{16}\right)$, it was simple to examine the parity of the coefficients of the power series for the new R.P.'s. Two of these possessed recognizable patterns which could be proved using Jacobi's triple product formula. In this theorem we will let $I=\{n \in \mathbf{N}: n \equiv \pm 1, \pm 2, \pm 5, \pm 6, \pm 8, \pm 9(\bmod 20)\}$ and $J=\{n \in \mathbf{N}: n \equiv \pm 2, \pm 3, \pm 4, \pm 5, \pm 6, \pm 7(\bmod 20)\}$.

\section{THEOREM 7.1.}

$$
\prod_{n \in I} \frac{1}{1-x^{n}} \equiv 1+\sum_{n=1}^{\infty}\left(x^{n(5 n-3) / 2}+x^{n(5 n+3) / 2}\right) \quad(\bmod 2)
$$

and

$$
\prod_{n \in J} \frac{1}{1-x^{n}} \equiv 1+\sum_{n=1}^{\infty}\left(x^{n(5 n-1) / 2}+x^{n(5 n+1) / 2}\right) \quad(\bmod 2) .
$$

Proof of (18). We have $(\bmod 2)$ that

$$
L=\sum_{n=-\infty}^{\infty}(-1)^{n} x^{n(5 n+3) / 2} \equiv 1+\sum_{n=1}^{\infty}\left[x^{n(5 n-3) / 2}+x^{n(5 n+3) / 2}\right]
$$

so by Theorem 355 in [8, p. 284],

$$
\begin{aligned}
L & \equiv \prod_{n=0}^{\infty}\left(1+x^{5 n+1}\right)\left(1+x^{5 n+4}\right)\left(1+x^{5 n+5}\right) \\
& =\prod_{n=0}^{\infty} \frac{\left(1+x^{5 n+1}\right)^{2}\left(1+x^{5 n+4}\right)^{2}\left(1+x^{5 n+5}\right)^{2}}{\left(1+x^{5 n+1}\right)\left(1+x^{5 n+4}\right)\left(1+x^{5 n+5}\right)} \\
& \equiv \prod_{n=0}^{\infty} \frac{\left(1+x^{10 n+2}\right)\left(1+x^{10 n+8}\right)\left(1+x^{10 n+10}\right)}{\left(1+x^{5 n+1}\right)\left(1+x^{5 n+4}\right)\left(1+x^{5 n+5}\right)} .
\end{aligned}
$$

But

$$
\prod_{n=0}^{\infty} \frac{\left(1+x^{10 n+10}\right)}{\left(1+x^{5 n+5}\right)}=\prod_{n=0}^{\infty} \frac{1}{\left(1+x^{10 n+5}\right)}
$$


so

$$
\begin{aligned}
L & \equiv \prod_{n=0}^{\infty} \frac{\left(1+x^{10 n+2}\right)\left(1+x^{10 n+8}\right)}{\left(1+x^{5 n+1}\right)\left(1+x^{5 n+4}\right)\left(1+x^{10 n+5}\right)} \\
& =\prod_{n=0}^{\infty} \frac{\left(1+x^{10 n+2}\right)^{2}\left(1+x^{10 n+8}\right)^{2}}{\left(1+x^{10 n+2}\right)\left(1+x^{10 n+8}\right)\left(1+x^{5 n+1}\right)\left(1+x^{5 n+4}\right)\left(1+x^{10 n+5}\right)} \\
& \equiv \prod_{n=0}^{\infty} \frac{\left(1+x^{20 n+4}\right)\left(1+x^{20 n+16}\right)}{\left(1+x^{5 n+4}\right)\left(1+x^{5 n+1}\right)\left(1+x^{10 n+2}\right)\left(1+x^{10 n+8}\right)\left(1+x^{10 n+5}\right)}
\end{aligned}
$$

But

$$
\prod_{n=0}^{\infty} \frac{\left(1+x^{20 n+4}\right)}{\left(1+x^{5 n+4}\right)}=\prod_{n=0}^{\infty} \frac{1}{\left(1+x^{20 n+9}\right)\left(1+x^{20 n+14}\right)\left(1+x^{20 n+19}\right)}
$$

and

$$
\prod_{n=0}^{\infty} \frac{\left(1+x^{20 n+16}\right)}{\left(1+x^{5 n+1}\right)}=\prod_{n=0}^{\infty} \frac{1}{\left(1+x^{20 n+1}\right)\left(1+x^{20 n+6}\right)\left(1+x^{20 n+11}\right)},
$$

which imply that $L \equiv \prod_{n \in I} 1 /\left(1-x^{n}\right)$.

The proof of (19) is similar, using Theorem 356 in [8].

Remark. There are infinitely many modular results obtainable from special cases of the Jacobi triple product formula by the method used above. We will give here the simplest one, the well-known result, derived from Theorem 353 in [8]:

$$
\prod_{n=0}^{\infty} \frac{1}{1-x^{2 n+1}} \equiv \sum_{n=-\infty}^{\infty} x^{n(3 n+1) / 2} \quad(\bmod 2) .
$$

An outstanding problem in this direction is to find a simple rule that gives the parity of the partition function $p(n)$, possibly using two or more products $(\bmod 2)$, whose parity conditions are known, and whose product is $\Pi_{n=1}^{\infty} 1 /\left(1-x^{n}\right)$. (See [12].)

8. Combinatorial Interpretations. The well-known combinatorial interpretations of the Rogers-Ramanujan identities (pairs 3 and 4 in Table 1) can be extended to the other six Ramanujan pairs (5-10 in Table 1). In order to list these partition interpretations concisely, we introduce the following notation. Fix $r=3, \ldots, 10$ and let $\left\{a_{i}\right\},\left\{b_{j}\right\}$ be the $r$ th Ramanujan pair listed in Table 1. We then define $p_{r}(n)$ and $q_{r}(n)$ by

$$
\prod_{i=1}^{\infty}\left(1-x^{a_{i}}\right)^{-1}=\sum_{n=0}^{\infty} p_{r}(n) x^{n}
$$

and

$$
1+\sum_{j=1}^{\infty} x^{b_{j}} /(x)_{j}=\sum_{n=0}^{\infty} q_{r}(n) x^{n}
$$

Of course, $p_{r}(n)=q_{r}(n)$. The interest lies in the interpretation of $p_{r}(n)$ and $q_{r}(n)$. That of $p_{r}(n)$ is obvious from the product form of (21). For example, $p_{5}(n)$ enumerates the number of partitions of $n$ into parts congruent to $\pm 1, \pm 4, \pm 6$, or $\pm 7(\bmod 16)$. To interpret $q_{r}(n)$, write a partition of $n$ in the form $n=c_{1}+c_{2}$ $+\cdots+c_{m}$, where the $c_{k}$ 's are in decreasing order of magnitude, i.e., $c_{k} \geqslant c_{k+1}$. In 
general, the results for the various cases impose minimal difference restrictions on certain of the $c_{k}$ 's, as summarized in the following

TheOREM 8.1. Let $\left\{a_{i}\right\},\left\{b_{j}\right\}$ be the rth Ramanujan Pair listed in Table 1. Then, $q_{r}(n)$ enumerates the partitions $n=c_{1}+c_{2}+\cdots+c_{m}$ satisfying the following conditions:

$$
\begin{array}{lll}
r=3: & c_{k}-c_{k+1} \geqslant 2, & 1 \leqslant k \leqslant m-1 ; \\
r=4: & c_{k}-c_{k+1} \geqslant 2, & 1 \leqslant k \leqslant m-1, \text { and } c_{m} \geqslant 2 ; \\
r=5: & c_{2 k}-c_{2 k+1} \geqslant 2, & 1 \leqslant k \leqslant \llbracket \frac{m-1}{2} \rrbracket, \text { and if } m \text { is even, } c_{m} \geqslant 2 ; \\
r=6: & c_{2 k}-c_{2 k+1} \geqslant 2, & 1 \leqslant k \leqslant \llbracket \frac{m-2}{2} \rrbracket, c_{m} \geqslant 2, \text { and if } m \text { is odd }, \\
r=7: & c_{m-1}>c_{m} ; & \\
r=8: & c_{2 k-1}>c_{2 k+1}, & 1 \leqslant k \leqslant \llbracket \frac{m-1}{2} \rrbracket ; \\
r=9: & c_{2 k-1}>c_{2 k}, & 1 \leqslant k \leqslant \llbracket \frac{m}{2} \rrbracket, \text { and if } m \text { is odd }, c_{m-1}>c_{m} ; \\
r=10: & c_{2 k}>c_{2 k+1}, & 1 \leqslant k \leqslant \llbracket\left[\frac{m-2}{2}\right], \text { and } c_{m} \geqslant 2 .
\end{array}
$$

When $r=3$ and 4, Theorem 8.1 gives us the familiar interpretations of the Rogers-Ramanujan identities. As discussed in Hardy and Wright [8, pp. 290-291], the translation of $q_{2}(n)$ into partition theory is based on the identity $b_{n}=n^{2}=1+$ $3+\cdots+(2 n-1)$, while that of $q_{4}(n)$ uses the equation $b_{n}=n(n+1)=2+4$ $+\cdots+2 n$. Following the method in [8], the results in Theorem 8.1 for the other cases are obtained by writing the exponents $b_{2 n-1}$ and $b_{2 n}$ for each R.P. as a sum of $2 n-1$ and $2 n$ terms, respectively. Thus, for $r=5$, we have

$$
\begin{aligned}
b_{2 n-1} & =2 n^{2}-1 \\
& =1+3+3+\cdots+(2 n-3)+(2 n-3)+(2 n-1)+(2 n-1)
\end{aligned}
$$

and

$$
b_{2 n}=2 n(2 n+1)=2+2+\cdots+(2 n-2)+(2 n-2)+(2 n)+(2 n),
$$

while the proof for $r=10$ uses

$$
b_{2 n-1}=n(n+1)=2+2+3+\cdots+(n-1)+(n-1)+n+n
$$

and

$$
b_{2 n}=n(n+3)=2+2+3+\cdots+n+n+(n+1)+(n+1) .
$$

Observe that the combinatorial interpretation for the Hirschhorn R.P. $(r=5)$ is a generalization of the Rogers-Ramanujan theorems in that a minimal difference of 2 is required of every other pair of terms and minimal part 2 is required only when there is an even number of terms in the partition. 
The interpretation in Theorem 8.1 for $r=8$ first appeared as B. Gordon's Theorem 7 in [7], while the result for $r=7$ is equivalent to W. Connor's Proposition 4 [5]. In that same paper, Connor gives different partition interpretations for $r=9$ and 10 than those in Theorem 8.1 [cf. Propositions 2 and 3]. The results for identities $r=5$ and 6 , as far as we know, are new.

Department of Mathematical Sciences

Northern Illinois University

DeKalb, Illinois 60115

Department of Mathematics

University of Arizona

Tucson, Arizona 85721

Department of Applied Mathematics and Statistics

SUNY at Stonybrook

Stonybrook, New York 11794

1. D. Acreman, Asymptotic Analysis of Partition Identities, Ph.D. Thesis, University of New South Wales, 1983.

2. H. L. Alder, "The nonexistence of certain identities in the theory of partitions and compositions," Bull. Amer. Math. Soc., v. 54, 1948, pp. 712-722.

3. G. E. ANDrews, “An incredible formula of Ramanujan,” Austral. Math. Soc. Gaz., v. 6, 1979, pp. 80-89.

4. R. Blecksmith, The Determination of Ramanujan Pairs, Ph.D. Thesis, University of Arizona, 1983.

5. W. ConNor, "Partition theorems related to some identities of Rogers and Watson," Trans. Amer. Math. Soc., v. 214, 1975, pp. 95-111.

6. L. EulER, Introductio in Analysin Infinitorum, Marcum Michaelem Bousquet, Lousannae, 1748, Chapter 16.

7. B. Gordon, "Some continued fractions of the Rogers-Ramanujan type,” Duke Math. J., v. 32, 1965, pp. $741-748$.

8. G. H. HARDY \& E. M. WRIGHT, An Introduction to the Theory of Numbers, 4th ed., Oxford Univ. Press, 1965.

9. M. D. Hirschhorn, "Two further Ramanujan pairs,” J. Austral. Math. Soc. Ser. A, v. 30, 1980, pp. $1-4$.

10. D. H. Lehmer, “Two nonexistence theorems of partitions," Bull. Amer. Math. Soc., v. 52, 1946, pp. 538-544.

11. J. LePOWSKy \& S. MiLne, "Lie algebraic approaches to classical partition identities," $A d v$. in Math., v. 29, 1978, pp. 15-79.

12. T. R. Parkin \& D. Shanks, "On the distribution of parity in the partition function," Math. Comp., v. 21,1969 , pp. $466-480$.

13. L. J. Slater, "Further identities of the Rogers-Ramanujan type," Proc. London Math. Soc. (2), v. 54, 1952, pp. 147-167. 